\title{
Microplastical microplastiche
}

\author{
Raffaella Setti
}

PUBBLICATO: 18 MAGGIO 2020

$\mathrm{M}$ ai forse come in questi ultimi mesi di emergenza sanitaria planetaria per il COVID-I9, abbiamo toccato tanto da vicino quanto siano estese, gravi e non più rimandabili le conseguenze ambientali del modello di sviluppo globale basato sul massimo e indiscriminato sfruttamento delle risorse del pianeta e sul conseguente accumulo di una quantità di rifiuti che ci stanno divorando, non solo sommergendoci e occupando spazi che prima erano esclusivi della natura (foreste, mari, deserti), ma anche entrando in modo "invisibile" nella catena alimentare e arrivando così a colpirci dall'interno.

La recente storia della parola microplastica e del suo referente- sembra aver seguito un percorso simile, con una diffusione "invisibile", non solo nel significato, di cui forse la maggior parte di noi non è ancora pienamente consapevole, ma anche nella sua forma; sembra messa in dubbio l'esistenza stessa della parola e la sua appartenenza al nostro lessico, visto che nessun dizionario italiano contemporaneo per adesso l'ha registrata.

Del problema dello smaltimento e del riutilizzo della plastica si parla ormai da decenni, ma solo dalla fine del Novecento si è scoperta questa nuova "forma" in cui può presentarsi la plastica: frammenti piccolissimi, prodotti dalla lentissima decomposizione di oggetti più grandi, che tendono a raccogliersi in vortici negli oceani e, in generale, nelle acque. Tonnellate e tonnellate di plastica che soffocano la vita del pianeta.

Tutto ha inizio con un incontro casuale: nel 1997 l'oceanografo Charles Moore, di ritorno da una regata tra Los Angeles e le Hawaii, nel nord del Pacifico si imbatté in quella che poi è stata denominata Great Pacific Garbage Patch (la grande isola di plastica del Pacifico), una sterminata discarica galleggiante di rifiuti plastici di varie dimensioni, da bottiglie, tappi, involucri fino a frammenti. Già nel 1999 Moore ha denunciato che in quella parte dell'oceano c'era una quantità di plastica sei volte maggiore rispetto allo zooplancton, alimento vitale per l'oceano stesso. Il racconto di questa scoperta e delle ricerche degli anni immediatamente successivi si ritrova nel suo volume Plastic Ocean (201I, uscito in traduzione italiana, L'Oceano di plastica, per Feltrinelli nel 2013). Da quel primo "incontro" le isole di plastica, o meglio vortici capaci di attrarre e risucchiare materiale di ogni tipo, sono diventate cinque, una per ciascun oceano più alcune più piccole individuate nel Mediterraneo. Si sono estesi gli studi, si è delineata una terminologia e ha cominciato ad essere usata, diffusa e meglio conosciuta la parola microplastica/che.

Il termine italiano per indicare questi residui microscopici di materiale plastico è un calco dall'inglese microplastic(s), registrato nell'Oxford English Dictionary come sostantivo (di seguito a microplastic, adj.) con attestazioni fin dal 1990: dagli anni '8o del secolo scorso, infatti, sono documentati studi sulla quantità di frammenti plastici presenti nei mari e negli oceani sulla base del loro depositarsi sulle spiagge, progressivamente sempre più consistente. Anche in inglese, in particolare in questa prima fase di diffusione, il termine presenta alcune varianti: in primo luogo è sia singolare sia plurale, microplastic e microplastics, e questo è dovuto al fatto che, ancor prima di assumere la funzione nominale per identificare l'insieme dei frammenti plastici inquinanti, indica, come aggettivo, ciò che designa o si riferisce a, o è causato da una deformazione plastica a un livello interno, microscopico, che si verifica 
tipicamente nelle sollecitazioni al di sotto del punto di deformazione irreversibile del materiale; altre varianti sono micro plastic, micro-plastic, micro plastics e micro-plastics, rintracciabili in rete, anche se ormai decisamente minoritarie rispetto alla forma prevalente microplastics, ma che non lasciano dubbi sulla modalità di formazione del termine, composto dal confisso micro- 'piccolo' (dal greco mikrós 'piccolo') e dal sostantivo plastic(s) 'plastica/(-che)' e ne rivelano la progressiva affermazione che ha portato, dalla forma con i due formanti staccati, passando per la variante con trattino, alla definitiva univerbazione.

L'italiano microplastica/che è un calco dall'inglese, di cui riflette significato e varianti formali. Partiamo dal significato che si è andato costruendo e precisando nel corso degli ultimi anni in parallelo con le ricerche, sempre più numerose e approfondite, che hanno avuto come oggetto questa tipologia di rifiuti. Per avere una definizione del termine, visto che nessun dizionario italiano lo registra, possiamo partire dalla voce microplastiche dell'Enciclopedia Treccani online: "particelle di materie plastiche, prodotte direttamente o indirettamente dall'uomo, le cui dimensioni sono state convenzionalmente fissate dall'European food safety authority tra o, I e 5000 micrometri'. Aggiungo che l'indicazione convenzionale delle dimensioni dei frammenti fissata dall'Efsa risale all'in maggio 2016 (Presence of microplastics and nanoplastics in food, with particular focus on seafood, efsa.onlinelibrary.wiley.com II/5/2016) e che anche un'altra autorevole fonte, l'ECHA (European Chemicals Agency), ha definito la microplastica "un insieme di particelle solide estremamente piccole (in genere di dimensioni inferiori a $5 \mathrm{~mm}$ ) composte da miscele di polimeri (i componenti principali della plastica) e da additivi funzionali". Successive precisazioni hanno portato a distinguere tra microplastiche primarie, prodotte come tali sotto forma di granuli o microsfere, e microplastiche secondarie, effetto dei processi di degradazione dei rifiuti di plastica di maggiori dimensioni. Per quel che riguarda la forma della parola e le sue varianti, osserviamo un andamento del tutto analogo a quello dell'inglese, con un periodo di oscillazione e compresenza delle varianti micro plastica, microplastica, micro plastiche e micro-plastiche, che, nel corso degli ultimi Io anni, tendono a convergere nelle due forme univerbate, il collettivo microplastica e, ormai prevalente, il plurale numerabile microplastiche.

Le prime attestazioni della parola in italiano, in questa accezione, si hanno tra il 20Io e il 20II: in particolare la prima che sono riuscita a rintracciare in rete è contenuta in un testo pubblicato sulla Gazzetta ufficiale dell'Unione Europea del $\mathrm{I}^{\mathrm{o}}$ settembre 20iо:

Tendenze nella quantità, nella distribuzione e, se possibile, nella composizione di microparticelle (in particolare microplastiche (Io.r.3). Io.2. Impatti dei rifiuti sulla vita marina (Decisione della Commissione europea del $1^{\circ}$ settembre 2010 sui criteri e gli standard metodologici relativi al buono stato ecologico delle acque marine, pubblicata sulla Gazzetta ufficiale dell'Unione Europea del 2/9/2oro).

Senza dubbio, in ambito scientifico e nella comunicazione istituzionale internazionale, il termine ha circolato dal 1990 al 20ro, in inglese e, in ambito europeo, nelle altre lingue dell'Unione, ma le prime apparizioni nella stampa italiana si hanno, in modo sporadico e molto rarefatto, solo dal 20II/20I2, con un'incidenza molto più consistente, a conferma della conquista di uno spazio nella lingua comune, tra il 2017 e il 20I9. Se si interrogano gli archivi dei principali quotidiani nazionali, il primo passaggio di microplastiche lo si trova in un articolo uscito sul quotidiano "La Stampa" del i8/ıo/20II:

[...] Il filo conduttore della prima parte del documentario è proprio il progetto di ricerca condotto dalla professoressa Maria Cristina Fossi e dal suo gruppo di ricercatori, uno dei pochi progetti al mondo che sia incentrato sugli effetti delle microplastiche - pezzetti di plastica di dimensioni sotto ai $5 \mathrm{~mm}$ - sul plancton ed in particolare sui grandi cetacei come la Balenottera comune. ("Plastic Oceans", le microplastiche e i loro effetti sulle balene, laspampa.it, Io/ro/20II) 
La notizia mette insieme le ricerche sul fenomeno e il progetto di realizzare un documentario (A Plastic Ocean, diretto dal giornalista australiano Craig Leeson con la partecipazione di ricercatori, fondazioni e persone comuni, uscito poi nel 20I6), ma dall'inizio dello stesso anno erano state molte le occasioni di studio e comunicazione scientifica. L'in marzo 20I si scatena lo tsunami in Giappone, un evento catastrofico che, oltre alla terribile devastazione e all'enorme quantità di vittime, contribui a portare "a galla", visibili all'umanità intera, le dimensioni e la gravità del problema dell'inquinamento da plastiche e microplastiche. Nello stesso anno si tengono importanti convegni scientifici internazionali dedicati ai detriti marini e alle conseguenze sulla fauna; in particolare, sempre a marzo 20II, a Honolulu, si svolge la Quinta Conferenza internazionale sui detriti marini in cui scienziati e biologi si confrontano sull'impatto delle microplastiche sulla fauna: se ne trova un tempestivo resoconto in un articolo del 4 aprile zor sulla rivista Terra Nuova:

Le plastiche sono materiali di lunga durata e restano nell'ambiente per centinaia d'anni (le cosiddette plastiche «biodegradabili» si frammentano solo più in fretta). E nuovi studi scientifici - citati sempre a Honolulu - mostrano come le microplastiche possono rilasciare sostanze chimiche che disturbano i sistemi endocrini, minacciando la salute delle specie marine. (La plastica nei mari uccide, terranuova.it, $4 / 4 / 201 \mathrm{I})$

Anche in Italia, nello stesso anno, il IX Congresso della Società italiana di biologia marina (Olbia 2328 maggio 2ori) dedica molto spazio al problema e uno studio è rivolto nello specifico alla questione dell'Ingestione di microplastica da parte di anfipodi sopralitorali (A. Ugolini e G. Ungherese):

Introduzione - La presenza di vari tipi di plastica in mare e lungo i litorali è un fatto evidente da molti
anni (Barnes et al., 2009; Thompson et al., 2009). Soltanto recentemente, tuttavia, è stata considerata la
relazione fra presenza di microplastica (frammenti < Imm) in ambiente marino, possibilità di ingestione
e suoi possibili effetti su organismi appartenenti ai livelli più bassi della piramide alimentare.
Microplastica è stata ritrovata in numerosi organismi acquatici appartenenti a vari taxa, fra questi
alcuni molluschi, anellidi policheti, zooplancton, crostacei (Thompson et al., 2004; Teuten et al., 20og).
(A. Ugolini, G. Ungherese, Ingestione di microplastica da parte di anfipodi sopralitorali, Biologia Marina
Mediterranea, 2oII, p. 396).

Da queste prime attestazioni del termine si deduce che l'analisi di questi piccolissimi residui e del loro impatto sull'ambiente è da subito connesso agli studi sulla fauna marina (e poi acquatica in senso lato) e condotta parallelamente a quella sul plancton e, in generale, sui microorganismi che normalmente vengono ingeriti da alcune specie animali. E nel 20r3, al momento dell'uscita della traduzione italiana del libro di Moore, il tema viene rilanciato attraverso la stampa in forme più divulgative:

L'isola della spazzatura il Settimo Continente che minaccia il Pacifico che cita il libro L'Oceano di plastica: «spessa zuppa di plastica, una zuppa abbondantemente condita con fiocchi di plastica, con l'aggiunta qua e là di pezzi di plastica grossi come boe, gomitoli di rete, galleggianti, cassette e altri detriti più consistenti». «Non è soltanto un danno estetico, si tratta di una minaccia per la vita marina che si fa di anno in anno più grave», ricorda Katrin Schroeder, oceanografa del Cnr di Venezia. «Tartarughe e delfini si impigliano nelle reti abbandonate, che li avvolgono in una trappola mortale. E poi c'e l'impatto sui pesci e sugli uccelli acquatici che ingeriscono plastica fino ad esserne soffocati. Purtroppo ancora non conosciamo la dimensione esatta di questo fenomeno, servono più informazioni sulle concentrazioni di microplastiche sospese nella colonna d' acqua» (Antonio Cianciullo, "La Repubblica", 28/o4/2013).

Di microplastiche si comincia a parlare anche in relazione ai fiumi (che trasportano rifuti fino ai mari) e addirittura ai laghi:

Non sono solo gli oceani a soffrire per l'inquinamento da materie plastiche: ora anche i laghi sembrano 
esserne colpiti. È la preoccupante scoperta di un team di ricercatori svizzeri che ha rilevato un'elevata concentrazione di microplastiche nel lago di Ginevra, lo specchio d'acqua più grande di tutta l'Europa occidentale (Rebecca Mantovani, I laghi alpini sono inquinati come i mari, focus.it, 30 maggio 2013, ).

E la microplastica diventa oggetto di ricerche anche nell'ambito dell'alimentazione, non solo per l'arrivo sulle nostre tavole del pesce che l'ha ingerita, ma addirittura per la sua presenza nel sale:

La microplastica che contamina pesci e molluschi (secondo un recente studio pubblicato su Scientific Reports in percentuali che si aggirano attorno al 30\% dei primi, e al 50-60\% dei secondi), si ritrova anche nel sale marino, almeno in Cina. L'hanno rilevata i ricercatori della Donghua University di Shanghai andando a verificarne la presenza nel sale normalmente venduto al supermercato. (Agnese Codignola, ilfattoalimentare.it, I7 novembre 20I5; fa riferimento a un articolo pubblicato su Nature nel settembre 2015)

Dunque oceanografi, geologhi e zoologi marini, scienziati ambientali e dell'alimentazione non solo mostrano il loro grande interesse per il fenomeno, ma investono risorse ed energie per portare all'attenzione pubblica internazionale $\mathrm{i}$ rischi e le conseguenze devastanti che si prospettano per l'intero pianeta. Da questo punto di vista i loro sforzi non sembrano essere stati sufficienti, almeno in Italia, a far circolare in modo ampio e capillare il "discorso" sulle microplastiche e, con esso- l'uso diffuso e comune della stessa parola microplastica/che: nelle pagine in italiano di Google, si contano 55.IOO occorrenze di microplastica e 249.000 di microplastiche (al 26/04/2020), numeri davvero contenuti per la rete.

Un quadro migliore è offerto dai principali quotidiani nazionali: dalla consultazione degli archivi del "Corriere della Sera", "La Repubblica" e "La Stampa" risulta un aumento significativo dell'impiego del termine a partire dal 2017 con una netta affermazione della variante microplastiche sul singolare collettivo microplastica: in particolare su "Repubblica" per microplastica si passa da 9 occorrenze nel 2016 a 2I nel 2017 con un incremento ulteriore nel 2018 (4I occorrenze) per poi notare un calo negli anni successivi dovuto al prevalere della forma microplastiche che, dalle 53 occorrenze del 20I7, arriva alle I42 del 20r9; un analogo andamento si registra nella "Stampa" con un incremento di occorrenze sia di microplastica nel 2017 (9 a fronte di una sola nel 2016) sia di microplastiche, che sale a 32 nel 2017 (nel 2016 ne aveva solo 6) per continuare ad aumentare negli anni successivi (44 del 20I8, 86 del 2019).

Tra questi articoli una sintesi particolarmente efficace è quella offerta da Mario Tozzi sulla "Stampa" del 27 aprile 20I7, che ripercorre la storia dalla scoperta di Moore nel Pacifico del nord fino alla denuncia del consumo esorbitante dei sacchetti della spesa e al paradosso per cui la plastica, materiale considerato indistruttibile e quindi simbolo dell'innovazione e della modernità, produce un enorme e pericolosissimo inquinamento ambientale proprio in virtù di questa sua ottima resistenza:

Però sono soprattutto sacchetti (shopping bag), di cui se ne fabbricano 500 miliardi all'anno (e pensare che nel 1970 nemmeno esistevano), che costituiscono circa il 40\% dei rifiuti marini del Mediterraneo, mangiati dalle tartarughe marine, che li scambiano per meduse, soffocando. [...]

Si tratta soprattutto di polietilene e polipropilene, ma anche di frammenti più pesanti come poliammidi e vernici, oltre a policaprolactone, un polimero considerato biodegradabile. Questa microplastica è costituita da frammenti più piccoli di 2 millimetri che, per quanto non visibili a occhio nudo, sono stati trovati a galleggiare pressoché ovunque nel Mediterraneo, con concentrazioni tra le più alte al mondo.

Nel vortice subtropicale del Pacifico settentrionale, nel 1999, sono stati stimati circa 335.0oo frammenti di plastica per chilometro quadro, mentre nel Mediterraneo si parla di una media di circa I,25 milioni. 
Nel tratto di mare tra la Toscana e la Corsica è stata rilevata la presenza di circa io chilogrammi di microplastiche per chilometro quadro, contro i circa 2 presenti a largo delle coste occidentali della Sardegna e della Sicilia e lungo il tratto nord della costa pugliese (dati Ismar-Cnr). [...]

Luomo inventa la plastica, un materiale a contenuto tecnologico incommensurabile rispetto a ognuno dei materiali naturali, e anche a quelli artificiali, fino a quel momento creati. È un materiale agile, che corre, vola e nuota. È straordinariamente resistente, ma ora abbiamo scoperto che si scioglie e si corrode, anche se solo in parte. E ha iniziato a rilasciare sostanze contaminanti a lungo termine. (Mario Tozzi, Ogni anno buttiamo nel mare 500 miliardi di sacchetti della spesa, "La Stampa", 27/04/2017).

In questo stesso arco di tempo (tra il 2017 e il 2019), il rischio rappresentato dalle microplastiche per la salute del pianeta, denunciato a più riprese da studiosi e ricercatori, inizia ad avere le prime concrete ripercussioni sulla vita dei cittadini europei e la parola entra con maggiore frequenza nel discorso pubblico e nei mezzi di comunicazione. I passaggi a più alto impatto mediatico si hanno tra il gennaio 2018 e il gennaio 2020. Già la Direttiva europea 720 del 2015 indicava ai paesi membri obiettivi di riduzione dell'uso dei sacchetti di plastica e di altri prodotti da cui si generano microplastiche (cosmetici esfolianti, bastoncini cotonati e particolari fibre tessili, ecc.). In Italia i primi effetti si hanno con la diffusione di sacchetti biodegradabili e borse riciclabili soprattutto nella grande distribuzione e poi, a partire dal I ${ }^{\circ}$ gennaio 20I8, con l'obbligo di far pagare anche i sacchetti ultraleggeri (biodegradabili o no).

Anche se danno un contributo modesto all'inquinamento, anche i sacchetti ultraleggeri oggetto della normativa sono una presenza che sporca $i$ mari, contaminati per esempio dalle temutissime microplastiche sviluppate soprattutto dalle fibre tessili rilasciate dalle lavatrici nei lavaggi e dal polverino di gomma degli pneumatici che si usurano sull'asfalto, ma sviluppate anche da cosmetici, bastoncini cotonati e altri rifiuti. I dati sull'inquinamento dei rifiuti nel mare sono riportati più sotto (Jacopo Giliberto, Ecco come funziona la legge sui sacchetti biodegradabili, ilsole24ore.com, 4/or/2018, ).

Nell'inverno 20I7-20I8 viene poi condotto uno studio sulla presenza di microplastiche nell'atmosfera e si comincia a parlare di microplastiche atmosferiche:

Uno studio, condotto dalle Università di Strathclyde in Scozia e di Orléans e Tolosa in Francia e da EcoLab di Tolosa durante l'inverno 2017-2018, va a colmare proprio questo deficit, studiando la deposizione di microplastiche atmosferiche in una zona montuosa remota, incontaminata e scarsamente abitata.

Lo studio ha quindi dimostrato come le microplastiche, trasportate dal vento, possono raggiungere e colpire aree remote, scarsamente abitate, dove non ci si aspetterebbe di trovare della plastica, almeno non in tali quantità. (Trovate microplastiche in aria, arpat.toscana.it, 28/05/2019)

Sempre nel 2018 l'allora Presidente della Commissione Ambiente della Camera Ermete Realacci riesce a far passare un emendamento alla Legge di Bilancio che prevede, a partire dal ${ }^{\circ}$ gennaio 2020 , il divieto di utilizzare microplastiche nei prodotti cosmetici da risciacquo. Si tratta di un provvedimento che coinvolge prodotti comuni, che tutti conoscono e usano (detergenti, scrub, dentifrici e prodotti esfolianti in genere), e che quindi contribuisce ulteriormente a far entrare nell'esperienza di molti il problema e la terminologia connessa:

Da gennaio 2020 è scattato il divieto alle microplastiche nei prodotti cosmetici da risciacquo

L'industria cosmetica utilizza, infatti, microplastiche come agente esfoliante o additivo in diversi prodotti di uso quotidiano. Ma l'emendamento non comprende tutti i prodotti cosmetici [...] a presenza di microplastiche in mare costituisce una minaccia grave per l'ambiente e per gli animali marini che, scambiandole per cibo, le ingeriscono, ma rappresenta un pericolo anche per gli esseri umani. Secondo L'Ispra, infatti, il $15-20 \%$ delle specie marine che finiscono sulle nostre tavole contengono 
microplastiche e solo in Italia, avverte "associazione ambientalista, una persona consuma in media circa 25 chili di pesce all'anno. (Addio alle microplastiche nei cosmetici, cosa c'e da sapere, adnkronos, 2/I/2020)

In definitiva, microplastica/che è una parola che, in più varianti, a partire dall'inglese microplastics, è presente in italiano ormai da vari anni (sicuramente dal 20Io) e la cui diffusione e progressivo radicamento hanno seguito, come avviene sempre per i termini coniati per definire qualcosa di nuovo, l'evolversi del fenomeno, le scoperte e gli studi che gli sono stati dedicati e l'ingresso del tema nella discussione pubblica. Limpulso in questa direzione è stato dato soprattutto dalle istituzioni europee che hanno preso atto dei risultati delle ricerche scientifiche e hanno emanato direttive e indicazioni rivolte ai paesi membri con l'obiettivo di ridurre il consumo di prodotti plastici, in particolare quelli maggiormente responsabili del rilascio di microplastiche (sacchetti monouso, prodotti detergenti leviganti e scrub). Dal punto di vista strettamente linguistico i testi emanati a livello europeo sono stati determinanti anche nel diffondere le traduzioni/calchi dall'inglese nelle altre lingue comunitarie, tra cui l'italiano microplastica/che. La stampa ha poi rilanciato il termine dalla comunicazione istituzionale alla lingua comune rendendo la parola (e il fenomeno) più conosciuta e utilizzata.

\section{Cita come:}

Raffaella Setti, Microplastica/microplastiche , "Italiano digitale", 2020, XIII, 2020/2 (aprile-giugno)

DOI: $10.35948 / 2532-9006 / 2020.3327$ 